AEDO. M. - GONZÁLEZ. V - FIERRO E. • Danzar el plural: hacia una experiencia afectiva y colaborativa de la danza como disciplina

\title{
Danzar el plural: hacia una experiencia afectiva y colaborativa de la danza como disciplina $^{1}$
}

Dancing the plural: towards an affective and collaborative experience of dance as a discipline

María Paz Aedo

Instituto de Estudios Avanzados (IDEA), UniversidAd DE SANTIAGO, CHILE. UNIVESIDAD DE DE BARCELONA, ESPAÑA. ${ }^{2}$

Vilma González

UNIVERSIDAD ACADEMIA DE HUMANISMO CRISTIANO, CHILE. ${ }^{3}$

Emilia Fierro

UNIVERSIDAD ACADEMIA DE HUMANISMO CRISTIANO, CHILE. ${ }^{4}$

Resumen. Las artes escénicas en general y la danza en particular, pueden entenderse como territorios dinámicos, espacios de encuentro y co-creación de mundos compartidos. El objetivo de este artículo es reconocer las trayectorías y fenómenos emergentes en los procesos de investigación realizados por tres docentes de la Escuela de Danza de una universidad. El enfoque metodológico de la investigación se desarrollará mediante autoenografías. El análisis de la investigación da cuenta de una perspectiva del cuerpo, no solo como unidad fisiológica susceptible de ser entrenada para representar una determinada coreografía o narrativa; sino como corporeidades, espacios plurales donde se encarna y actualiza nuestra experiencia de ser en el mundo, afectando y siendo afectado por multiplicidad de experiencias y vínculos. Como conclusión, la investigación identifica la danza como disciplina que puede movilizar afectos y corporeidades orientados a la colaboración y el diálogo democrático antes que a la transmisión de conocimiento experto.

Palabras clave: danza; corporeidades; pluralidad; formación; creación

Abtsract. The performing arts in general and dance in particular can be understood as dynamic territories, meeting spaces and co-creation of shared worlds. The objective of this article is to recognize the trajectories and emerging phenomena in the research processes carried out by three teachers from the Dance School of a university. The methodological approach of the investigation will be developed through autoenographies. The analysis of the investigation shows a perspective of the body, not only as a physiological unit capable of being trained to represent a certain choreography or narrative; but as corporeities, plural spaces where our experience of being in the world is embodied and updated, affecting and being affected by multiplicity of experiences and links. In conclusion, the research identifies dance as a discipline that can mobilize affects and corporations oriented to collaboration and democratic dialogue before the transmission of expert knowledge.

Key words: dance; corporeities; plurality training; creat

\footnotetext{
${ }^{1}$ Investigación realizada en el marco de los Fondos de Investigación y Creación (FIIC). Universidad Academia Humanismo Cristiano 2019-2020.

${ }^{2}$ Licenciada en sociologa Universidad de Chile. Doctora en educación Universidad Bolivariana. Postdoctorante Facultad de Artes y Educación. Universidad de Barcelina. Mail: maria.aedo.z@usach.cl

3 Licenciada en Danza Universidad Academia Humanismo Cristiano. Docente Universidad Academia Humanismo Cristiano: Mail: vilmagonzáleziturriaga@gmail.com

4 Licenciada en Danza y Magíster en Liderazgo Transformacional Universidad Academia Humanismo Cristiano. Docente Universidad Academia Humanismo Cristiano. Mail: emi.fciriza@gmail.com
} 


\section{Introducción}

En nuestra formación y deriva cultural, la racionalidad instrumental constituye una prioridad en el aprendizaje y está a la base de lo que se entiende como conciencia propiamente humana, mientras que los afectos y las corporeidades se entienden como "irracionalidades" que desbordan, incluso peligrosamente, dicha racionalidad. Tomando distancia de esta tradición, el "giro afectivo" de la filosofía y las ciencias sociales viene a recordarnos la condición encarnada y afectiva de nuestra condición vital, donde el afecto constituye fuerza, intensidad y capacidad para mover y ser movido (Cvetkovich 4; Braidotti et al., 13).

La experiencia de estar vivos es profundamente relacional y contextual. Todo lo que hemos aprendido a ser y estar siendo, tal como todo lo que observamos y hemos aprendido a observar, ocurre con otros y a través de otros. Desmontando el supuesto de realidad unívoca y externa, los enfoques post-humanistas y neo-materialistas proponen universos múltiples y realidades especulativas, donde los distintos agentes, humanos y no humanos, co-crean mundos desde su respectiva trayectoria vital, generando convergencias e intersecciones ${ }^{5}$.

Al reconocer esta multiplicidad, es posible tensionar las tradiciones educativas y creativas basadas en la reproducción sistemática y jerárquica del conocimiento experto, transitando hacia el diálogo de los distintos actores, considerando sus memorias, afectos y experiencias como saberes. Ello no supone, por cierto, un relativismo total y la imposibilidad de generar tradiciones o disciplinas. Más bien, se trata de generar espacios de formación y creación que reconozcan, concidiendo con Haraway, el carácter situado y contextual del conocimiento que promueven (30), en diálogo con la voz activa de estudiantes e intérpretes. La formación y creación disciplinar, por tanto, puede entenderse como ejercicio de actualización situada y dialógica de un área de conocimiento.

Como en toda disciplina, en la danza desembocan y son provocadas nuevas y múltiples interpretaciones (Strand 46). Así, podemos entender la danza como un fenómeno hermenéutico que nos afecta, movilizando, develando e interpelando nuestra manera de habitar y comprender el mundo. Que el "soporte" de esta disciplina sea el cuerpo no implica que se trate de un fenómeno irracional e intuitivo; ni tampoco estricta y unívocamente racional. Tal como señala Borgorff, esta confusión:

surge cuando el contenido no conceptual de los hechos artísticos se confunde

con su supuesta forma no cognitiva. Sin embargo, los fenómenos de trabajo

en el terreno artístico son decididamente cognitivos y racionales, aun cuando

no podemos acceder directamente a ellos a través del lenguaje y los conceptos

(23).

Tanto la reproducción mecánica de la técnica, como la exigencia de estándares insondables o difusos por parte de expertos/as, están relacionadas con la falta de reflexión sobre el proceso

\footnotetext{
${ }^{5}$ La dramaturga Manuela Infante, en su clase magistral "El teatro nunca ha sido humano" (2019), realizada en el marco de los laboratorios escénicos del Festival Santiago a Mil, ofrece una clara relación entre estos enfoques y las artes escénicas. Disponible en: https://teatroamil.tv/clases-magistrales/clase-magistral-manuela-infante/.
} 
AEDO. M. - GONZÁLEZ. V - FIERRO E. • Danzar el plural: hacia una experiencia afectiva y colaborativa de la danza como disciplina

de formación y creación. Reflexionar sobre los afectos (fuerzas, intensidades y movimientos) emergentes en la actualización disciplinar ofrece la posibilidad de transitar hacia procesos de formación y creación situados y dialogantes, que reconozcan y potencien las relaciones de influencia recíproca entre docentes y estudiantes, creadores e intérpretes.

El Magíster en Educación, Mención en Liderazgo Transformacional de la U. Academia de Humanismo Cristiano, ofreció en sus dos especializaciones (Gestión Escolar y Comunidades de Aprendizaje $)^{6}$ una experiencia de tutoría-acompañamiento basada en la investigación autoetnográfica. Este artículo recoge las trayectorias y fenómenos emergentes en los procesos de investigación realizados por tres docentes de la Escuela de Danza de esta Universidad, que al momento de la investigación se encontraban cursando el Magíster?

\section{Giro afectivo, corporeidades y entrelazamientos}

Frente a las limitaciones del pensamiento científico positivista y la "crisis de representación" de las ciencias sociales (Blanco 170), desde mediados de los años 80 y más claramente desde inicios de los 90, han emergido diversas maneras y formatos para la investigación y el aprendizaje en el campo social, humanista y artístico, conocidas como el "giro afectivo" (Lara 104). Este "giro" revela a cada participante no como un "yo" racional que habita un cuerpo irracional, y que es atravesado por emociones también irracionales, sino como un conjunto de corporeidades, entramados vitales habitados y constituidos por afectaciones e influencias recíprocas. La idea de un sujeto aislado en la particularidad de su "ser" desconoce la condición dinámica, histórica y relacional de su "estar siendo", generando tramas y nudos de recurrencia. Para Clark y Mcphie, la existencia humana:

no se desarrolla en lugares sino a lo largo de caminos. Procediendo a lo largo de

un camino, cada habitante establece un sendero. Donde los habitantes se

encuentran, los senderos se entrelazan, ya que la vida de cada uno se enlaza con

el otro. Cada entrelazamiento es un nudo, y cuanto más se entrelazan las líneas

de vida, mayor es la densidad del nudo. Los lugares, entonces, son como nudos,

y los hilos de los que están atados son líneas de viajeros (4).

La experiencia humana es, entonces, trayectoria y entrelazamiento, plural y no singular. Tal como podemos observar en la creación artística, la particularidad, e incluso la inconmensurabilidad de la perspectiva personal, no es sinónimo de singularidad. Para Blanco, "una vida individual puede dar cuenta de los contextos en los que vive la persona en cuestión, así como de las épocas históricas que recorre a lo largo de su existencia" (170). Lo aparentemente trivial y único se devela como "demostraciones de sabiduría y conocimiento

\footnotetext{
${ }^{6}$ La especialidad "Comunidades de Aprendizaje” se implementó una sola vez, entre los años 2018 y 2019. La especialidad "Gestión Escolar" ha tenido lugar desde 2014 a la fecha.

${ }^{7}$ La autora principal de este artículo se desempeñó como tutora e informante de estos tres docentes entre los años 2016 y 2019.
} 
sensible (...) que actúan en la paradoja de universalidad y particularidad" (Van Manen 136). El cuerpo también participa de esta dinámica y necesita ser considerado en su pluralidad, como liminalidad que emerge entre la experiencia individual y colectiva; esto es, como corporeidad. Para Pérez Royo, dicha noción "se refiere a los modos aprendidos y construidos del cuerpo en su estar, presentarse, relacionarse y moverse con otros" (13). No solo como un aprendizaje consciente, entrenado, sino como "derivadas del aprendizaje cultural y social dentro de una determinada geografía" (14).

En definitiva, el llamado "giro afectivo" ofrece un marco para transitar desde la centralidad del individuo, el lenguaje y la razón instrumental, hacia el reconocimiento de los afectos y corporeidades como pluralidades constitutivas de la experiencia humana. Al aceptar la condición plural de los afectos y corporeidades, la formación y la creación artística son también fenómenos políticos, marcados por una tradición disciplinar autoritaria, reducida a la transmisión de conocimiento experto y centrada en un ideal reificado del artista y su "deber ser" (Ramírez 86), arrojado a su propio e inconmensurable mundo. Dicha tradición convierte al arte en una experiencia elitizada, accesible solo a través del privilegio o del sacrificio. Es preciso, por tanto, transitar hacia una actualización disciplinar dinámica, situada y democrática que facilite el acceso de los y las actuantes a una experiencia de aprendizaje y creación simultáneamente introspectiva y colaborativa, enriquecida por las reflexiones y el diálogo de saberes entre los distintos actores involucrados. Si los cuerpos aprenden, pueden "encarnar diferentes corporalidades y si éstas son las que determinan cómo estamos juntos, tiene sentido pensar en un entrenamiento favorable al proyecto democrático" (Pérez Royo 16).

Así, danzar deja de ser solo el escenario de expertos y es un territorio de visibilización, diálogo y encuentro entre personas equivalentes, dispuestas a compartir, colaborar y coinspirarse, cada cual a su manera y según sus circunstancias.

\section{Investigar lo plural}

Cabe preguntarse cómo accedemos a la pluralidad de fenómenos presentes en la formación y creación de la danza, toda vez que no se reducen al mundo del lenguaje y la transmisión de conceptos. Hacerse cargo de estas preguntas supone tensionar la tradición moderna y racionalista del paradigma científico en la generación de conocimiento, caracterizada por una distinción dualista entre ejercicio práctico y ejercicio investigativo (Carillo 221; Sánchez 89). Para Hernández-Hernández, en los inicios del método científico, el empirismo y el positivismo:

(...) establecieron las bases del denominado método científico, se ha naturalizado una relación de carácter unívoca entre investigación científica e investigación (...) Esta visión de la investigación científica (...) ha marcado durante casi trescientos años al pensamiento occidental y ha significado, por ejemplo, aceptar como necesaria la separación entre el sujeto que observa e investiga y el objeto observado y sobre el que se investiga (87-88). 
AEDO. M. - GONZÁLEZ. V - FIERRO E. • Danzar el plural: hacia una experiencia afectiva y colaborativa de la danza como disciplina

Al considerar que la práctica docente y artística están inextricablemente implicadas en el "objeto" a investigar (la creación, el aprendizaje), este dualismo no se corresponde con la experiencia. Además, si se establece una relación activo-pasiva entre actores, se reducen las posibilidades de reflexión sobre el fenómeno artístico y educativo. Frente a las limitaciones del pensamiento científico positivista y la "crisis de representación" de las ciencias sociales (Blanco 170), desde mediados de los años 80 y más claramente desde inicios de los 90, han emergido diversas maneras y formatos para la investigación en el campo social y humanista. Estos nuevos enfoques pueden aunarse bajo la definición de "metodologías post-cualitativas" (Lather y St Pierre 629), que buscan relevar la voz protagónica del sujeto investigado como actor capaz de reflexionar e indagar sobre su experiencia; y al mismo tiempo, develar al investigador como actor no neutral ni externo, desde una perspectiva "emic" (Sánchez 99). El género metodológico post-cualitativo se ha desarrollado, principalmente, en el campo de las artes y las humanidades, para dar cuenta de "fenómenos sociales, artísticos y culturales más amplios" (Carrillo 231-232), según la narrativa de los actores involucrados.

Estos métodos pueden ubicarse dentro de diversos marcos teóricos: feministas, poscoloniales, fenomenológicos, antirracistas, deconstructivos y experimentales (Carrillo 231). Según Sánchez, algunos de ellos son:

el proyecto metodológico indigenista como metodología de descolonización;

la etnografía crítica; el humanismo crítico; la teoría queer; la etnografía performativa; los estudios narrativos; la investigación basada en el arte; la etnografía online; las poéticas cultuales, entre otras, las cuales se han situado en las fronteras entre el pospositivismo (...) y el posestructuralismo (...)

En coherencia con un enfoque performativo y agencial, lo investigado no constituye un dato observado para la construcción de conocimiento en el sentido convencional, sino que invita a la participación de todos los actores en su co-creación, estableciendo, tal como señala Hernández-Hernández:

(...) un nuevo nivel de relación fundamental: contar una historia que permita a otros contar(se) la suya. El objetivo no sería sólo capturar la realidad sino producir y desencadenar nuevos relatos. (Así abre), entre otras, las siguientes opciones: (a) dejar espacios que pueden ser 'llenados' por los diferentes lectores; (b) tratar de evitar la ficción perfecta que represente de manera unívoca la realidad; (c) hacer visible el metarrelato que brinde sentidos 
alternativos al trayecto investigador, y (d) dar la posibilidad al lector de completar el relato (97).

Este enfoque tensiona también el concepto de subjetividad presente en la tradición científica, que supone un individuo cuyo relato y experiencia personal es trascendido (y, desde la tradición estrictamente positivista, omitido) para construir conocimiento generalizable. De este modo, como afirma Giselda Hernández, la investigación postcualitativa ofrece pistas para transitar hacia "una clase sustentada, sobre la plataforma de la confianza entre los actores sociales involucrados" (41). Asimismo, permite abordar el problema que representa la singularidad del lenguaje académico y especialista, democratizando el acceso y abriendo posibilidades de participación, al servicio de los y las participantes: "la utilización de códigos culturales ampliamente compartidos y de imágenes populares hace que (...) sean más accesibles que lo que ofrece el habitual lenguaje académico o experto" (Hernández-Hernández 110).

Así, el ejercicio de indagar en las trayectorias afectivas y corporales de docentes, estudiantes, creadores e intérpretes implica participar de un entramado vital dinámico antes que naufragar en la búsqueda de certezas y datos. Más bien, buscamos señales, pistas, resonancias con lo que está pasando en el proceso de creación y aprendizaje, observando los afectos y los cuerpos que se movilizan y nos interpelan (Carrillo 234). Esto es relevante para la investigación en artes y educación, puesto que "el propio estudio, como cualquier otro tipo de investigación, es una empresa corporeizada" (Hernández-Hernández 110). El actorinvestigador se mueve y habita el mundo en influencia recíproca con lo investigado.

La investigación autoetnográfica es un tipo de investigación post-cualitativa que, en base a la observación de la propia experiencia, permite generar procesos reflexivos afines a las premisas antes descritas. La diferencia entre el relato autoetnográfico y autobiográfico se relaciona con el énfasis: en la autoetnografía, el actor habla "desde sí", reconociendo la trama dinámica que encarna, lo moviliza y atraviesa en forma no lineal y no "de ś́,", como un relato secuencial de vivencias individuales. Enfatiza la reflexividad de las experiencias propias como campos de investigación, conectando "las distancias entre yo y el otro (...) en un proceso en el que el autor hace públicos los mecanismos de su propia labor, en un continuo descubrimiento o desvelamiento, que supone a la vez un esfuerzo académico y una performance cultural" (Hernández-Hernández 107). Ofrece la oportunidad de dar sentido a lo vivido, atrayendo "nuestros sentidos, emociones e intelecto (descubriendo) lo que no sabíamos que sabemos, o lo que vemos que no nos habíamos dado cuenta antes, incluso cuando está presente frente a nosotros" (Hernández Hernández 108).

La fortaleza de la autoetnografía como práctica reflexiva es reconocida por Suárez como "un suelo fértil para el pensamiento y la acción pedagógicos" (764), donde cada docente se constituye como un actor protagónico en la construcción de saberes pedagógicos para la "auto y co-formación de docentes y de intervención discursiva en el campo político y público de la educación" y que "necesariamente, implican indagaciones y producción de saber y la formación inicial y permanente de los docentes” (Suárez 771).

Gracias a la observación recursiva o "meta-observación" de su experiencia y de los mundos creados a partir de ellas, el/la investigador/a se despoja progresivamente de su "deber ser" para reconocerse como un actor que está siendo lo que ha aprendido a ser (Aedo et al. 
AEDO. M. - GONZÁLEZ. V - FIERRO E. • Danzar el plural: hacia una experiencia afectiva y colaborativa de la danza como disciplina

3). El ejercicio autoetnográfico, simultáneamente introspectivo y dialógico, contribuye significativamente a la construcción participativa de conocimiento, reduciendo la tendencia a la mediación explicativa. Tal como señala Saldívar et al., propicia "la reflexión y el análisis sobre la propia práctica educativa (...) como un espacio donde se explicitan los elementos pedagógicos vivenciados en las actividades" (114).

El rigor metodológico de la autoetnografía está sostenido por la auto-reflexión crítica; esto es, que "ambas partes (la teórica y la práctica) se complementen y se informen la una a la otra, y que el proceso de producción sea autoreflexivo, es decir, que se cuestione a sí mismo" (Carrillo 223). De esta manera, es posible transitar desde la separación tradicional entre investigador y objeto de estudio, orientado al conocimiento "puro", hacia un involucramiento del docente/creador como investigador/observador del proceso en que está activamente involucrado, orientado al conocimiento situado, al diálogo de saberes y a la profundización democrática de la práctica disciplinar. Se trata, por tanto, de una práctica que permite a los y las participantes de la danza no renunciar al rigor en aras de una subjetividad relativista -donde "todo vale"-, sino experimentar la actualización disciplinar como ejercicio, donde la particularidad es acogida e integrada en lo plural, de manera dialogante y contextualizada.

En coherencia con estos enfoques, el Magíster en Liderazgo Transformacional y Gestión Escolar/Comunidades de Aprendizaje ha promovido procesos de investigación autoetnográfica de manera transversal al período formativo (18 meses continuos). Estas investigaciones, tutorizadas por un/a docente del Magíster en calidad de aprendiz y observador de los fenómenos emergentes, se han sostenido en tres procesos paralelos: elaboración de bitácora personal mensual; reuniones periódicas de acompañamiento individual y grupal y elaboración de reflexión personal al término de cada módulo temático (8 en total). En estos tres procesos, se invita a los y las estudiantes a dar cuenta de su experiencia vivida con énfasis en los "lugares" (memorias, corporeidades y afectos) desde donde emergían sus observaciones e interpretaciones. Adicionalmente, cada tutor/a ofrece su mirada a sus acompañados/as, explicitando también desde dónde escuchaba, observaba e interpretaba, tensionando las fronteras convencionales de maestro-aprendiz.

A partir de la experiencia de acompañamiento y elaboración de informes finales llevada a cabo con docentes de la Escuela de Danza que participaron como estudiantes del Magíster, es posible observar sus exploraciones, hallazgos, reflexiones y aperturas de su "ser profesional", así como los fenómenos emergentes y las actualizaciones/acciones realizadas a propósito de sus procesos reflexivos, como se expone a continuación.

\section{Del "yo" al "nosotros": tensiones y controversias en la composición colectiva}

El proyecto de danza "Matriz" surge de un laboratorio de investigación autoetnográfica que Vilma Iturriaga, docente de eukinética, coreógrafa y estudiante del Magíster, llevó a cabo con un equipo de intérpretes, en colaboración con el equipo de trabajo de la escuela Matríztica que coordinan Humberto Maturana y Ximena Dávila. La creación estuvo centrada en observar y manifestar la condición humana como fenómeno emergente y colectivo, asumiendo que en la cualidad y textura de los movimientos es posible advertir la condición entramada de cada actor y su corporeidad. Desde este enfoque, la obra fue concebida como una experiencia afectiva, donde, en el deseo de mover y ser movido, cada participante actualizara -más que "representara"-su experiencia vital. 
Durante cuatro meses, el proyecto consistía en la realización de ejercicios de reflexión y experimentación sobre la trayectoria de cada participante, revisitando, actualizando y encarnando sus memorias, saberes y afectos. La propuesta invitaba a los y las participantes a revisitar el origen, particularmente la experiencia de separación implicada en el proceso de individuación; y actualizar/encarnar preguntas vitales: ¿cómo construir un "nosotros", a partir de la diferenciación?; ¿cómo habitar, simultáneamente, la particularidad de cada experiencia y el deseo de convergencia?; ¿cómo articular la diversidad de corporeidades deseantes en torno a un proyecto compartido?

Con este fin, el proceso de creación inició con un "conversar liberador", espacio de diálogo orientado a la exploración de cada participante con el sentido de sí mismo y sus experiencias de conexión. A partir de estas conversaciones, los y las participantes elaboraron cartografías que dieran cuenta de las emergencias, utilizando diferentes materialidades, dibujos y texturas. Sobre esta base -el relato y la creación emergente-, exploraron movimientos, acompañados de musicalidades y sonidos favorables a la intimidad. Sin embargo, al poco andar, la indagación de cada participante y su puesta en común para la construcción de un movimiento colectivo, generó simultáneamente afectos potenciadores del encuentro (apertura, reconocimiento) y limitantes (desconfianzas, miedos). Esta tensión se vinculaba directamente con la relación entre la propuesta y la tradición disciplinar moderna descrita al inicio de este artículo. Mientras que la tradición ofrece espacios de aprendizaje basados en la problematización y búsqueda de respuestas, con miras a reducir la incertidumbre, el proceso de Matriz proponía revisitar las preguntas sin buscar respuestas; sin intencionar la búsqueda de perfección ni soluciones de continuidad. Se requería, por tanto, aprender a habitar la incertidumbre, llanos a la emergencia.

$\mathrm{Al}$ reconocer que estas afectaciones recaían sobre Vilma en su condición de creadora de esta obra, la tutoría se centró en la búsqueda de movimientos que contribuyeran a sostener la paradoja del yo-nosotros, sin buscar necesariamente una solución de continuidad. A partir de los registros autoetnográficos y las conversaciones de acompañamiento, Vilma y su tutora acordaron explorar la estabilidad y la exploración de límites como desafíos de aprendizaje personal y colectivo. Así, descubrieron la posibilidad de habitar la estabilidad como espacio liminal, fenómeno emergente en el desplazamiento de un estadio vital a otro y de un movimiento a otro; y los límites como bordes dinámicos, que invitaban a la revisión de estándares y la negociación cotidiana en aras del cuidado de cada participante y del conjunto, más que como imposiciones derivadas del "deber ser" propios de pedagogías convencionales y de la necesidad de control.

Sobre esta base, Vilma aportó al equipo de Matriz un espacio de confianza favorable al tránsito por la incertidumbre y al diálogo sobre los límites móviles de la creación. Así, cada participante llevó a cabo el ejercicio de revisitar sus memorias y experiencias, identificando qué o cuáles eran los acontecimientos que estaban más presentes, si querían o podían re-significarlos, cuál era la mirada hoy de esa historia, qué querían traer y contar, y de qué manera (Gutiérrez 12). Todas esas reflexiones y preguntas fueron llevadas a ejercicios de improvisación. El relato personal encarnado en movimientos dio lugar a la creación de una narrativa corporal de sus vivencias, donde sin aspirar a resolver las tensiones, sino más bien habitándolas, fue posible co-crear una puesta en escena dinámica, donde cada intérprete podía recrear libremente lo que deseaba/necesitaba expresar. Según Gutiérrez: 
AEDO. M. - GONZÁLEZ. V - FIERRO E. • Danzar el plural: hacia una experiencia afectiva y colaborativa de la danza como disciplina

En medio de las tensiones, me di cuenta que lo que para algunos era una obra, para mí era la oportunidad de crear una nueva manera de mirar y habitar el mundo, más allá de la obra misma. Es muy posible que esta diferencia de miradas estuviera a la base de las contradicciones dentro del proceso. Si bien el resultado nos dejó en paz y resultó consecuente con el proceso vivido, en lo personal me doy cuenta que dentro del proceso mismo, particularmente en el mundo artístico, este es un camino por construir (...) podemos encontrarnos, sí; incluso podemos sabernos, en una dimensión profunda, entramados unos con otros. Y esto no significa, necesariamente, alineación de la observación y las acciones. Podemos discrepar y al mismo tiempo, llegar a resultados conjuntos; aunque no sean exactamente lo previsto por cada uno de nosotros por separado. Aunque podemos elegir lo que creamos y las posibilidades que queremos habitar, no somos plenamente conscientes ni estamos en control de todo lo que puede suceder cuando lo hacemos y cuando nos encontramos. En último término, la comunión con otros ocurre junto a nuestras inconmensurables miradas del mundo. La incertidumbre, entonces, también es mía (33).

La creación fue presentada en el Centro Cultural Gabriela Mistral durante una temporada de 16 funciones. En la última, tuvo lugar un conversatorio entre intérpretes, directora, el público asistente, Humerto Maturana y Ximena Dávila (Guitérrez 36).

Escuchar. Ver. Sentir. Dejar aparecer. Entregarse a otro. Darse. Siento felicidad (...) ¡Si tuviera menos edad! (...) Abrazaré a mi señora (...) Entregarme. Ser feliz. Evocaciones liberadoras. Sólo se precisa coraje. Gracias por el atrevimiento. Gracias por compartir con tanta generosidad el 
sentir y vivir que están viviendo. Muy honesto y mucho respeto a ustedes mismos (Gutiérrez 36-37)

Finalmente, los comentarios del público fueron recogidos por escrito. Esos relatos dan cuenta del movimiento afectivo y la resonancia que desde lo múltiple fue posible generar entre esta circunstancial comunidad de artistas y espectadores.

\section{Sin miedo al error: corporeidades y técnica académica}

La técnica académica, cátedra relacionada con el entrenamiento coreográfico de la danza clásica, supone un conjunto de movimientos estándares y normativas disciplinantes sobre el cuerpo y el movimiento. La técnica, así entendida, constituye un dispositivo de control sobre las corporeidades y genera resistencias micropolíticas, expresadas como irritación, frustración, conflictividad, ausentismo, etc. (Nilo 8). En este escenario, dos de los docentes que forman parte del cuerpo de profesores responsable de la formación en técnica académica y que realizaron una investigación autoetnográfica en el contexto del Magíster, reconocieron los afectos que generaba este conjunto de normativas en su experiencia como aprendices y profesionales de la danza. Específicamente, la sensación de insuficiencia y la presión por el logro de resultados.

A partir de la acogida a estos afectos "tristes" o despotenciadores, en los términos de Spinoza (Teles 118), el docente de técnica académica y estudiante del Magíster, Gabriel Nilo, observa un "punto ciego" o una "zona oscura" poco abordada en los diseños curriculares: el problema del sobre-entrenamiento, causado por entrenar sin dar tiempo suficiente a la recuperación. Durante el proceso de acompañamiento e investigación autoetnográfica ${ }^{8}$, para Nilo fue posible reconocer el que este problema se relaciona con la presión por encarnar el movimiento "correcto" que supone la técnica; problema que vivenciaba él mismo cotidianamente como docente y bailarín profesional. Los síntomas observables de este fenómeno no refieren solo al cuerpo fisiológico. El sobre-entrenamiento crea cuerpos lesionados, irritados y enfermos, que afectan los vínculos, las planificaciones, las normas y, en general, la estructura completa que conforman bailarines y bailarinas, profesionales y aprendices. Sin embargo, este fenómeno parece no responder a un patrón regular; es decir, no ocurre en fechas previas a exámenes o presentaciones, sino de forma aleatoria (Nilo 8).

Esta aleatoriedad permite afirmar que el sobre-entrenamiento emerge como una constante en el aprendizaje de la técnica académica, al ser concebida como sinónimo de "ballet" (Nilo 9). En tanto "ballet", la técnica predispone a los y las participantes a la obligación de sobre-exigirse y sobrecargarse para encajar en sus estándares y normas. La tensión entre la diversidad de corporeidades y los estándares asociados al ballet generan problemas de ausentismo, frustración y conflictividad (Nilo 8). Adicionalmente, la docente Emilia Fierro, también estudiante del Magíster y miembro del equipo docente responsable de la formación en técnica académica de la Escuela de Danza, da cuenta, en su investigación

\footnotetext{
${ }^{8}$ Tutorizado por la docente Soledad Rodríguez.
} 
AEDO. M. - GONZÁLEZ. V - FIERRO E. • Danzar el plural: hacia una experiencia afectiva y colaborativa de la danza como disciplina

auto etnográfica, de la tensión que genera la técnica académica como "ballet", por su tradición excluyente y elitista.

A partir de sus reflexiones y su propia inquietud como docentes de esta cátedra, los docentes Nilo y Fierro decidieron explorar otras posibilidades afectivas en la relación con sus estudiantes. Incorporaron dispositivos orientados explícitamente al cuidado y la escucha de las corporeidades implicadas, bajo las premisas de observar, distinguir y auxiliar los movimientos (Nilo 15-16) en vez de "corregir el error", dando tiempo y espacio al proceso de aprendizaje como tránsito entre distintos movimientos (Fierro 8). De esta manera, ofrecieron a cada participante, y a sí mismos, la posibilidad de reconocerse y desplazarse entre distintas posibilidades de actuación como parte del proceso de aprendizaje. La erradicación del "error" y la valoración de las diversidades permitió a los docentes ofrecer la técnica como un conjunto dinámico y apropiable de disposiciones al movimiento, que puede ser entrenada a través de la resonancia de los cuerpos y la recurrencia de los movimientos. Para ello, Nilo (2019) sostiene que es posible ofrecer al menos cuatro tipos de feedback:

Hay estudiantes que agradecen la explicación más física o biomecánica posible de dar (feedback descriptivo), mientras que para otros/as puede ser la imagen (feedback de imagen), tanto la metáfora sobre lo que se busca como la imagen del profesor/a mostrando lo que está pidiendo. También hay otros alumnos que para ciertas ejecuciones es de gran ayuda ser tocados en sus cuerpos (feedback táctil), mientras que otros necesitan una modificación en su contexto para realizar la acción de forma más eficaz (feedback contextual) (17).

La estrategia de escuchar y dialogar entre distintas corporeidades dio resultados. Al comparar los procesos de estudiantes de 2017 y 2018, Nilo observa una mejora en la calidad de la ejecución:

El segundo año, con menor volumen de sesiones en el total del entrenamiento de técnica académica, presentó a final de año la misma cantidad de repertorio de pasos que el tercer año (...) con mayor calidad, prolijidad y estabilidad en cada movimiento. Es decir, si un nivel menor, con menor cantidad de entrenamiento, alcanzó la misma cantidad de contenidos que un nivel mayor con el mismo docente a cargo de la cátedra, pero con mayor calidad de 
ejecución en el total del curso, se podría decir que (estas) estrategias podrían ser una herramienta útil y a considerar para la enseñanza de la técnica académica en un contexto de educación superior (25).

Por su parte, Fierro destacó la importancia del feedback afirmativo y no correctivo, que permita a cada participante "(...) ver qué camino ha recorrido y cómo lo ha hecho, así como también cómo recorrer lo que viene. A mí, como profesora, me permite lo mismo" (8). También, en coherencia con su propio proceso de instrospección y reflexión, la docente implementó al inicio de cada sesión prácticas de meditación, invitando a los y las estudiantes a detenerse y observar el movimiento desde la quietud, instalando la opción de habitar el cuerpo en reposo, atento y disponible a la experimentación de nuevas posibilidades. La respiración consciente emerge como espacio de liminalidad desde donde contemplarse.

Fierro llevó a cabo espacios de diálogo y análisis del proceso de aprendizaje con los y las estudiantes, en forma transversal a la cátedra: elaboración de mapas conceptuales, observación de los movimientos de la clase y contraste con las premisas de la cátedra "Análisis del movimiento" (10).

Es posible afirmar que las acciones realizadas han significado un avance significativo que comienza a entregar resultados muy sólidos en los estudiantes, potenciando el aprendizaje, el entendimiento y la apropiación de la técnica $(\ldots)$ hemos podido observar los buenos resultados con la comisión evaluadora del examen final y con algunos estudiantes. Las comisiones evaluadoras de segundo y tercer año entregaron muy buenos comentarios alusivos al trabajo de cohesión existente entre todos los participantes de cada grupo (41 y 20 estudiantes respectivamente), así como la solidez de su trabajo técnico. Los docentes encargados de evaluar mencionaron que, independiente de la presencia o ausencia de "destrezas" de ballet (piernas muy altas, exceso de elongación, entre otras), los cursos en general mostraban el dominio técnico pulcro y detallado que requiere la técnica académica en su esencia, y que permite a ésta complementar otros lenguajes y técnicas. A diferencia de años anteriores, en los cuales unos cuántos destacaban en los exámenes por 
AEDO. M. - GONZÁLEZ. V - FIERRO E. • Danzar el plural: hacia una experiencia afectiva y colaborativa de la danza como disciplina

sus capacidades físicas sobresalientes, este año todos lograron un nivel técnico parejo y óptimo para la continuidad de la técnica de ahí en adelante. Así como los profesores evaluadores, los estudiantes también reconocieron en su trabajo una mejoría notoria en la estabilidad de su técnica, lo cual se tradujo en seguridad y tranquilidad respecto a la clase y los contenidos. La gran mayoría destacó un antes y un después luego de las metodologías utilizadas en clase, sobre todo en el curso de segundo año (Fierro 12).

Finalmente, estas innovaciones también se tradujeron en mejoras del aprendizaje de la técnica, especialmente en términos del trabajo de conjunto, observándose la emergencia del "plural".

\section{Conclusión}

El objetivo planteado en esta investigación, la identificación de trayectorias y fenómenos emergentes en los procesos de investigación realizados por tres docentes de la Escuela de Danza de una universidad, nos invita a reflexionar sobre la disciplina en su tradición racionalista, ya que se encuentra asociada a la transmisión lineal de un conocimiento legitimado como "experto" por la comunidad de pares. Sin embargo, esta forma de entender el aprendizaje supone reducir el proceso a la relación vertical entre maestro y aprendiz, generando afectos despotenciadores y pérdidas de riqueza del mismo.

Como hemos podido observar, cuando el "maestro" se sumerge en un proceso de reflexión crítica como el que ofrece la auto-etnografía, consigue despojarse de los supuestos y exigencias implícitas al rol docente y creador en este sentido tradicional; y abrirse a posibilidades de innovación y co-creación que no restan rigor ni calidad al proceso; antes bien, lo enriquecen y potencian. Esto es lo que entendemos por actualización democrática de la disciplina: un espacio de aprendizaje que permita sostener y habitar simultáneamente la tradición y la innovación, la singularidad y la pluralidad, la estructura y la apertura. Habitar estas oscilaciones y paradojas permite construir espacios de escucha dialógica y democrática entre docentes, estudiantes, creadores e intérpretes, diluyendo las barreras que reducen y separan estos roles; y los estereotipos que confunden al actor con el rol, generando quiebres y rupturas.

La contribución de esta investigación corresponde a las experiencias aquí expuestas, las que han contribuido a generar modos de crear y aprender que permiten a cada actor reconocerse y escucharse, para desde allí reconocer y escuchar a otros, generando climas de confianza y respeto. Así, cada singularidad, integrada en un espacio colectivo rizomático, permite la emergencia del nosotros-plural, corporeidades abiertas, divergentes y convergentes. 
Por cierto, los casos que hemos expuesto no buscan convertirse en modelos replicables ni premisas generalizantes; antes bien, se trata de propuestas que surgen a partir del potencial creativo, democrático y transformador que supone comprender la danza como movimiento afectivo de corporeidades que sienten, crean y reflexionan juntas.

A modo de corolario, cabe destacar que las tendencias que buscan incluir la corporalidad y los afectos para el aprendizaje de otras disciplinas, y que utilizan las artes escénicas como una estrategia didáctica o complementaria, son necesarias, pero insuficientes para dar cuenta de los afectos y corporeidades como dimensiones centrales en los procesos de aprendizaje y socialización. Es preciso reconocer y potenciar la comprensión de lo colectivo como corporeidades en movimiento dialógico, y la danza como un territorio donde es posible aprender, experimentar y ejercitar este movimiento dialogante y democrático. En tiempos en que las múltiples manifestaciones de la crisis civilizatoria amenazan seriamente nuestra vida y nuestra convivencia, esta manera de entender el cuerpo y el movimiento constituyen una estrategia central de resistencia. Como bien afirmaba Pina Baush, es preciso bailar, o estaremos perdidos.

\section{Bibliografía}

Aedo, María Paz, Santiago Peredo y Colombina Schaeffer. "From an essential being to an actor's becoming: political ecology transformational learning experiences in adult education". Environmental Education Research 25.1 (2019): 33-45. Impreso.

Blanco, Mercedes. “¿Autobiografía o autoetnografía?” Revista Desacatos. 38. Enero-abril (2012): 169-178. Web. 21 marzo 2020.

Braidotti, Rosi y Hlavajova Maria, eds. Posthuman Glossary (Theory). Londres: Bloomsbury Academic, 2018. Impreso.

Borgdorff, Henk. "El debate sobre la investigación en las artes." Cairon: Revista de ciencias de la danza. 13 (2010). Web. 23 marzo 2020.

Carrillo, Perla. "La investigación basada en la práctica de las artes y los medios audiovisuales," Revista Mexicana de Investigación Educativa 20.64 (2015): 219-240. Impreso.

Clarke, David y Mcphie Jamie "From Places to Paths: Learning for Sustainability, Teacher Education and a Philosophy of Becoming." Environmental Education Research 22.7. (2016): 1002-1024. Impreso.

Cvetkovich, Ann. Depression: A public feeling. Durham Duke University Press, Durham \& London, 2012. Impreso.

Fierro, Emilia. La importancia de la comunicación en la transmisión de saberes. Construcción de pedagogía a partir del diálogo. Tesis de Magíster Universidad Academia de Humanismo Cristiano. Santiago, 2019. Impreso.

Fuentes, Ángel. El valor pedagógico de la danza. Valencia: Universidad de Valencia. 2006. Impreso.

Guitérrez, Vilma. Experiencia de aprendizaje transformacional en procesos creativos y formativos. Tesis de Magíster Universidad Academia de Humanismo Cristiano. Santiago, 2018. Impreso.

Haraway, Dona. Ciencia, cyborgs y mujeres: la reinvención de la naturaleza Valencia: Ediciones Cátedra, Universidad de Valencia e Instituto de la Mujer, 1991. Impreso. 
AEDO. M. - GONZÁLEZ. V - FIERRO E. • Danzar el plural: hacia una experiencia afectiva y colaborativa de la danza como disciplina

Hernández-Hernández, Fernando "La investigación basada en las artes. Propuestas para repensar la investigación en educación." Revista Educatio Siglo XXI 26. (2008): 85118. Web 20 marzo 2020.

Hernández, Giselda. "Cuando se despeja la bruma: investigación basada en arte, un análisis dirigido al aprendiz de brujo" Tercio Creciente Revista de Estudios en Sociedad, Artes y Gestión Cultural 8. (2015): 39-46. Web. 27 marzo 2020.

Lara, Alí y Giazú Enciso Domínguez. "El Giro Afectivo". Athenea Digital. 13.3. (2013). 101-119. Web 18 marzo 2020.

Lather, Patti y St. Pierre Elizabeth. "Post-qualitative Research" International Journal of Qualitative Studies in Education 26.6 (2013): 629-233. Impreso.

Nilo, Gabriel. Danza, cuidados para el aprendizaje. Tesis de Magíster Universidad Academia de Humanismo Cristiano. Santiago: 2019. Impreso.

Pérez Royo, Victoria. "Componer el plural: una introducción”. Componer en plural. Eds. Pérez Royo, Victoria y Diego Aguiló. Barcelona: Mercart de Les Flors, Institut del Teatre y Ediciones Polígrafa, 2016. 9-29. Impreso.

Ramírez, Karina. "Aporte del ejercicio de la meta observación y el enfoque de la performatividad a la formación teatral." Revista Actos 2 (2019): 81-92.

Saldívar, Antonio, Moramay, Miriam, Santos Elizabeth y Ávila, Rocio. "Los retos en la formación de maestros en educación intercultural: La experiencia de La Casa de la Ciencia en Chiapas." Revista Mexicana de Investigación en Educación 9.20 (2004): 109-128. Web. 25 marzo 2020.

Sánchez, Zulma. "Investigación en educación artística. Más allá de los riesgos, la búsqueda por las posibilidades." Revista Pensamiento, Palabra y Obra 18 (2017): 87-100. Web. 2 marzo 2020.

Suarez, Daniel. "Espacio (auto)biográfico, investigación educativa y formación docente en Argentina: un mapa imperfecto de un territorio en expansión. Revista Méxicana de Investigación Educativa.Jun.2014.Web.23 abr.2020.

Strand, Dennis. Research in the Creative Arts. Evaluations and Investigations Program Report, 98/6. Canberra: Department of Employment, Education, Training and Youth Affairs, 1998. Impreso.

Teles, Annabel. Política afectiva: Apuntes para pensar la vida comunitaria. Paraná: Fundación La Hendija, 2009. Impreso.

Van Manen, Max. Investigación educativa y experiencia vivida: ciencia humana para una pedagogía de la acción y la sensibilidad. Barcelona: Idea-Books, colección Idea Universitaria- Educación, 2003. Impreso.

Recibido: 30 de marzo de 2020.

Aceptado: 16 de junio de 2020. 